\title{
O PROCESSO DE CONSTRUÇÃO DO CURRÍCULO NO MESTRADO PROFISSIONAL EM SAÚDE DA FAMÍLIA
}

\author{
THE PROCESS OF BUILDING THE CURRICULUM IN THE PROFESSIONAL MASTER'S DEGREE \\ IN FAMILY HEALTH
}

EL PROCESO DE CONSTRUCCIÓN DEL CURRÍCULO EN LA MAESTRÍA PROFESIONAL EN SALUD

DE LA FAMILIA

Maria de Fátima Antero Sousa Machado ${ }^{1}$

Neiva Francenely Cunha Vieira ${ }^{2}$

Virginia Alonso Hortale ${ }^{3}$

Anya Pimentel Gomes Fernandes Vieira-Meyer ${ }^{4}$

Ana Patrícia Pereira Morais ${ }^{5}$

Andréa Silvia Walter de Aguiar ${ }^{6}$

Annatália Meneses de Amorim Gomes ${ }^{7}$

Geórgia Sibele Nogueira da Silva ${ }^{8}$

Resumo Trata-se de uma reflexão sobre o processo de desenvolvimento do currículo do curso de Mestrado Profissional em Saúde da Família, oferecido pela Rede Nordeste de Formação em Saúde da Família. Foram atores desse processo docentes de instituições de ensino e pesquisa, federais e estaduais. A construção do currículo, com duração de dois anos, incluiu a formação do Grupo de Desenvolvimento Curricular, responsável pelo desenho do curso e pela formação dos seus docentes. Discutem-se as etapas desse processo tendo como referência as bases pedagógicas que nortearam o desenho do currículo, com ênfase na formação de profissionais da saúde na modalidade mestrado profissional. Espera-se que a divulgação dessa experiência inovadora no âmbito da formação stricto sensu contribua para que outras experiências possam não somente ser pensadas e implementadas, mas também divulgadas.

Palavras-chave formação em saúde; desenho de currículo; saúde da família.

\author{
Liberata Campos Coimbra ${ }^{9}$ \\ Maria Socorro de Araújo Dias ${ }^{10}$ \\ Maristela Inês Osawa Vasconcelos ${ }^{11}$ \\ Carlos Otávio Fiuza Moreira ${ }^{12}$ \\ José Batista Cisne Tomaz ${ }^{13}$ \\ Franklin Delano Soares Forte ${ }^{14}$ \\ Claudete Ferreira de Souza Monteiro ${ }^{15}$
}

Abstract This is a reflection on the process of developing the curriculum for the Professional Master's Degree in Family Health course offered by Northeast Training in Family Health Network. Professors from federal and state educational and research institutions participated in this process. The construction of the curriculum, that took two years to be completed, included the creation of the Curriculum Development Group, which was put in charge of designing the course and training its professors. The steps of this process were discussed with reference to the educational bases that guided the curriculum design, with emphasis on training health professionals in the professional master's degree mode. It is hoped that the dissemination of this innovative experience within the scope of stricto sensu training will contribute to other experiences not only being designed and implemented, but also disclosed.

Keywords training in health; curriculum design; family health. 


\section{Introdução}

Com a instalação de uma nova unidade da Fundação Oswaldo Cruz (Fiocruz) no Ceará e a criação da Rede Nordeste de Formação em Saúde da Família (RENASF), em 2010, inicia-se o processo de desenvolvimento da proposta de um curso em nível stricto sensu para profissionais vinculados à Estratégia Saúde da Família (ESF) da região Nordeste (Hortale et al., 2015).

Essa proposta insere-se na atual política da Coordenação de Aperfeiçoamento de Pessoal do Ensino Superior (Capes), que lançou em 2009 a Portaria n. 17, de 28 de dezembro de 2009 (Brasil, 2009), a qual explicita que o Mestrado Profissional deve "estimular a formação de mestres profissionais habilitados para desenvolver atividades e trabalhos técnico-científicos em temas de interesse público".

A definição de estrutura e funcionamento para sua criação deu-se com a participação de docentes de três tipos de instituições definidas pela RENASF, a saber: nucleadoras, colaboradoras, consorciadas. Nucleadoras são aquelas que tenham pelo menos um curso de pós-graduação stricto sensu na área da saúde, docentes para atuar nessa proposta, infraestrutura e que realizem procedimentos de certificação. Colaboradoras disponibilizam infraestrutura e docentes. Consorciadas apoiam as atividades de ensino e disponibilizam infraestrutura. Em relação ao credenciamento de docentes, observou-se a portaria n. 17/2009 MEC/Capes, no art. $7^{\circ}$, parágrafos 1 e 2, que tratam da qualificação e da produção científica e reconhecimento profissional (Brasil, 2009).

Outro aspecto relevante nesse processo foi a criação de uma rede colaborativa entre as instituições de forma descentralizada. A construção de uma rede de formação significa buscar sinergias e maximizar a funcionalidade das suas ações por meio das diferentes contribuições que cada membro da rede pode oferecer. Ocorre mediante a partilha de recursos e de infraestrutura, a mobilidade de docentes e alunos no interior da rede e a padronização dos planos de curso e sistemas de avaliação, mantendo, no entanto, as especificidades de cada componente da rede com vistas a acrescentar outras experiências ao conjunto (Santos, 2010).

$\mathrm{O}$ curso atende às políticas de educação permanente e à formação de profissionais dos serviços do Ministério da Saúde, com destaque à divulgação das produções científicas oriundas da realidade dos processos de trabalho nos diversos níveis da atenção. Tem como prioridade fortalecer a formação técnico-científica do profissional de saúde inserido na Atenção Primária, incentivando-o ao uso do método e de evidências científicas para a tomada de decisões na gestão do cuidado e do processo de trabalho. E, não menos importante, fortalecer a produção do conhecimento das instituições associadas à RENASF.

Dada a complexidade dos problemas sociais e a ampliação do conceito de saúde, o curso pretende aprofundar a compreensão do papel das relações 
sociais e de trabalho na determinação do processo saúde-doença-cuidado e na concepção ampliada de saúde, com suas consequências para a prática na ESF. A multiprofissionalidade e a intersetorialidade se apresentam como estratégias potentes no compartilhamento de saberes voltados à promoção da qualidade de vida.

Entendemos que experiências como esta devem ser disseminadas e, nesse sentido, cumprimos aqui o papel de divulgar uma experiência que pode contribuir com a elaboração de outras propostas de formação stricto sensu, modalidade mestrado profissional.

Relatamos neste artigo, à luz da literatura, a experiência de dois anos do processo de desenvolvimento do currículo do curso de Mestrado Profissional em Saúde da Família (MPSF), oferecido pela RENASF, e fazemos uma reflexão crítica sobre os limites e alcances de uma experiência em rede, no contexto brasileiro. Concentramos nossa narrativa na construção do currículo, desde a instituição do Grupo de Desenvolvimento Curricular (GDC).

\section{Concepção teórica da proposta do Mestrado Profissional}

A valorização dos conhecimentos voltados para os níveis de atenção de maior complexidade, como parte das estruturas curriculares dos cursos de graduação em saúde, vem sendo tratada sob forma plural.

A formação de profissionais de saúde tem gerado discussões cada vez mais recorrentes, nas quais se incluem o questionamento sobre aqueles currículos que não proporcionam conhecimentos advindos do exercício da problematização, da reflexão e da análise da realidade; um dos argumentos é que as demandas de saúde são complexas e requerem profissionais com capacidade para intervir na promoção da saúde individual e coletiva, considerando os determinantes sociais.

Conhecer cada contexto em que se estuda é o foco na produção de cuidado em saúde da população, seja a partir da análise do processo de trabalho em saúde, seja por meio das formas de adoecimento e sofrimento humano, ou ainda estas articuladas com as políticas de saúde, em especial as do Sistema Único de Saúde (SUS) (Binz, Menezes Filho e Saupe, 2010).

Ao tecer considerações sobre os processos formativos na área da saúde, não podemos esquecer que há excessiva ênfase nos aspectos clínicos do cuidado, fato gerador de pouco valor formativo para atenção primária em saúde (Barroso, Vieira e Varela, 2002; Saupe et al., 2007).

As Diretrizes Curriculares Nacionais, aprovadas entre 2001 e 2004 para os Cursos da Área da Saúde, vêm conferir um novo perfil de identidade ao ensino superior em saúde ao propor currículos mais articulados com a realidade social e que valorizam a concepção ampliada de saúde, o enfoque na 
promoção da saúde, a interdisciplinaridade, a qualidade de vida e o desenvolvimento de competências. Entretanto, as fronteiras para migrar ou construir entrelaçamentos entre teoria e prática ainda são frágeis, principalmente aquelas voltadas à promoção da saúde humana (Saupe et al., 2007).

Em meio a múltiplos olhares, surgem propostas que pensam um currículo com base em competências para atender uma formação contextualizada para atenção primária à saúde. Currículo este articulado às exigências da sociedade, aos conhecimentos, à preparação científica e à capacidade de utilizar diferentes tecnologias, de pesquisar, criar, formular, analisar e aprender a aprender nas condições em que se desenvolve o cuidado. Tudo isso significa que as competências cognitivas e culturais, essenciais para o desenvolvimento humano, podem influenciar efetivamente, na dimensão dialógica, a discussão produtiva durante o processo formativo e contribuir para o bem-estar dos indivíduos, famílias e comunidades (Frenk et al., 2010).

Um desenho curricular ajustado à realidade do mundo do trabalho na saúde é fundamental para o sucesso de um curso, independentemente da sua abordagem educacional (Kelly, 1981; Tomaz, 2001).

O currículo deveria contemplar, no dizer de Morin (2001), elementos científicos e humanísticos. Na formação em saúde, o currículo necessitaria incorporar a produção de subjetividades, as habilidades técnicas de pensamento e os conhecimentos adequados ao SUS. Assim, com base na problematização da realidade social, as práticas profissionais e a própria organização do trabalho se transformam, e passam a englobar o acolhimento e o cuidado nas várias dimensões e necessidades de saúde da população (Ceccim e Feuerwerker, 2004).

Construímos o currículo do Mestrado sob os pilares das competências profissionais para atuar na promoção da saúde individual e coletiva. Adotamos a definição de Zafirian (2001) para o termo 'competência', onde os conhecimentos, o saber fazer, as experiências e os comportamentos são postos para resolver uma situação em um determinado contexto. Com essa definição dinâmica exige-se, além da capacidade de ter iniciativas e responsabilidades, uma inteligência prática capaz de mobilizar outras competências na resolução de problemas ou situações.

Para o processo de desenvolvimento do desenho curricular privilegiamos alguns aspectos, a saber: a relação teoria-prática, a produção do conhecimento, o processo saúde-doença e as dimensões da promoção da saúde. Na relação entre a teoria e a prática busca-se integrar o conhecimento à ação, numa interdependência de trocas e reciprocidades, constituindo um todo único (Souza, 2001). Já a produção do conhecimento individual é aqui considerada os saberes, as habilidades e as atitudes do aluno como ser único que, quando compartilhado e integrado no grupo, resulta no conhecimento coletivo. O processo saúde-doença é entendido numa perspectiva ampliada 
considerando os determinantes biológicos, sociais e culturais dos indivíduos e da coletividade (Smith, Tang e Nutbeam, 2006).

\section{Caminho na elaboração do desenho do currículo}

O curso, aprovado pela Capes em outubro de 2010, contou com a participação de seis instituições de Educação Superior: Fundação Oswaldo Cruz, Universidade Federal do Ceará, Universidade Estadual do Ceará, Universidade Federal do Maranhão, Universidade Federal do Rio Grande do Norte e Universidade Estadual Vale do Acaraú.

Para a elaboração do currículo criamos o GDC, composto por representantes das instituições nucleadoras e instituições colaboradoras, com experiência na formação de profissionais da ESF. Um desses representantes assumiu a coordenação pedagógica.

Para assegurar essa proposta e que atendesse às concepções que favorecessem a formação com base na teorização-problematização-reflexão-ação, o GDC organizou três oficinas para a elaboração do currículo. Na primeira oficina abordou-se a formação por competências e discutiram-se os princípios do processo de ensino-aprendizagem, bem como o desenho do currículo baseado em competências. Na segunda, apresentou-se o conceito e as raízes históricas da aprendizagem baseada em problemas, iniciando-se a elaboração dos módulos do curso; e, por fim, na terceira oficina, apresentou-se a proposta da avaliação por competências.

Essas oficinas nos deram as condições para subsidiar a elaboração do que foi chamado 'matriz de competências' do MPSF, discriminando as atividades educacionais a serem adotadas.

O sucesso dessas oficinas possibilitou-nos estender essa formação para os demais docentes que participariam do curso, o que permitiu maior uniformização metodológica entre os docentes, a discussão e a construção coletiva das experiências de aprendizagem a serem conduzidas quando da implementação do curso. Assim, cada membro do GDC foi 'facilitador' do Curso de Atualização em Educação das Profissões da Saúde, do qual participaram 54 professores de cerca de 15 instituições.

Realizamos essa capacitação sob a forma de quatro oficinas, com carga total de 54 horas. Demos ênfase às temáticas da aprendizagem de adultos, suas implicações para o campo da saúde e as bases para elaboração de currículos baseados em competências.

Na concepção Problematizadora ou Ativa, adotada nesta proposta, o estudante é visto como ser ativo e de relações, e o papel docente passa a ser mediar o processo de aprendizagem do aluno, pois entende que o conhecimento é construído pelo sujeito na sua relação com os outros e com o mundo. O 
conteúdo precisa ser trabalhado, refletido, re-elaborado pelo aluno, para se constituir em conhecimento dele (Vasconcellos, 1992).

Ancorados nas metodologias ativas, adotaram-se, para a organização das situações de ensino aprendizagem, as estratégias educacionais, que são técnicas usadas para o alcance dos objetivo de aprendizagem. Anastasiou e Alves (2004) advertem que as estratégias visam à consecução de objetivos; portanto, há que ter clareza sobre aonde se pretende chegar naquele momento com o processo de ensinagem.

Bordenave e Pereira (1998) consideram estratégias de ensino como sendo um caminho escolhido ou criado pelo professor para direcionar o aluno, pautado numa teorização a ser aplicada na sua prática educativa. Para Masetto (2003), as estratégias de ensino e aprendizagem são meios utilizados pelo professor para facilitar o processo de aprendizagem dos alunos.

No currículo deste curso de MPSF, as estratégias educacionais foram adotadas para mobilizar os objetivos de aprendizagem propostos em cada módulo.

\section{Organização acadêmico-administrativa do curso}

Na construção do currículo do curso, um dos produtos desse processo foi a criação da coordenação acadêmico-administrativa do curso, atualmente sob a responsabilidade de um colegiado composto pelos coordenadores do curso das instituições nucleadoras.

Para fins de atender às exigências da Capes, o curso organizou-se na área de concentração Saúde da Família e em três linhas de pesquisa. No nosso entender, essa área de concentração, dada sua natureza complexa, compõe tanto uma área do conhecimento quanto um campo de práticas profundamente imbricado com as mudanças do modelo de atenção à saúde no Brasil, e demanda indissociabilidade entre teoria e prática e retroalimentação de referenciais teórico-metodológicos e político-institucionais na definição desses processos e na gestão, educação na saúde e produção do conhecimento desenvolvido no SUS.

Nessa perspectiva, adotamos os seguintes objetos de produção do conhecimento: saúde como direito e consequente necessidade de identificar e atender às demandas de saúde da população na perspectiva da humanização do cuidado; promover a saúde e dar importância estratégica ao desenvolvimento das ações intersetoriais e de fomento à participação popular e controle social; promover a integralidade do cuidado, a qual implica o trabalho em rede e equipe multiprofissional; promover a educação permanente e a gestão participativa dos serviços de saúde. Também privilegiamos o desenvolvimento profissional para aprimorar processos institucionais em nível locorregional, a partir do princípio da territorialização da atenção à saúde e que subsidiam a ESF. 
Esses objetos estão contemplados nas três linhas de pesquisa propostas. Na linha Atenção e gestão do cuidado em saúde, propusemos a formulação e o desenvolvimento de investigações com foco na elaboração, implantação e avaliação de iniciativas, projetos, programas e políticas de atenção e gestão do cuidado no contexto da ESF. Na linha Educação na saúde, propusemos a formulação, implantação e avaliação de iniciativas, projetos, programas e políticas de formação e desenvolvimento profissional dos trabalhadores de saúde, visando à reorientação de seus processos de trabalho. E na linha Promoção da saúde propusemos a formulação, implantação e avaliação de projetos e programas, com base nos determinantes da saúde, para promover o desenvolvimento de tecnologias inovadoras visando à melhoria das condições de saúde das populações, e fomentar a participação popular e a autonomia dos sujeitos no cuidado à saúde.

Definimos como objetivos do curso: formar lideranças para exercer atividades de investigação e de ensino em serviço, sem se afastarem de suas atividades na atenção ou gestão da ESF; fomentar o trabalho em equipe por meio do diálogo entre profissionais da equipe básica e dos Núcleos de Apoio à Saúde da Família (NASF); e articular elementos da educação, atenção, gestão e investigação no aprimoramento da ESF e do SUS, com ênfase na atenção primária.

\section{Matriz do curso}

O curso contempla uma estrutura curricular comum oferecida em rede, descentralizada, com predominância da utilização de metodologias ativas de ensino-aprendizagem. Em sintonia com a proposta teórica assumida pelo colegiado, o ponto de partida foi a elaboração das competências necessárias a um mestre em saúde da família, que em resumo são capacidade de:

- realizar ações de promoção da saúde;

- atuar na estratégia saúde da família na perspectiva da integralidade e humanização da atenção básica;

- utilizar métodos científicos para elaboração de projetos de investigação ou intervenção;

- desenvolver atividades de educação em saúde no contexto da atenção primária;

- produzir e utilizar informações em saúde na atenção primária;

- desenvolver projeto de investigação ou de intervenção como requisito para o trabalho de conclusão do curso;

- realizar a atenção e a gestão do cuidado do indivíduo, família e comunidade;

- realizar a gestão do processo de trabalho na estratégia saúde da família;

- realizar ações de planejamento e avaliação na atenção primária em saúde;

- desenvolver atividade de preceptoria nos serviços de saúde. 
A 'matriz de competências' é composta pelos objetivos de aprendizagem, estratégias educacionais e de avaliação. Adotou-se para organização do currículo a estrutura modular, compreendida de 11 módulos: Seminário de Introdução ao Curso; Promoção da Saúde; Atenção Integral à Saúde da Família; Produção do Conhecimento em Serviços de Saúde; Sistemas de Informação no Cuidado e na Gestão; Educação na Saúde; Avaliação na Atenção Primária; Gestão do Processo de Trabalho na Atenção Primária; Tópicos Especiais em Saúde da Família e Seminário de Acompanhamento I, II, III e IV; e a Dissertação.

As atividades de cada módulo compuseram o Caderno do Mestrando, elaborado pelo conjunto de docentes que participariam de cada módulo durante as oficinas de preparação do material didático. A utilização do Caderno teve por objetivo subsidiar o processo ensino-aprendizagem dos mestrandos e o acompanhamento dos docentes-facilitadores. Acreditamos que desse modo poderia haver maior uniformidade na condução do curso em cada nucleadora.

\section{Metodologias do curso}

Inicialmente, julgamos importante tratar da concepção de método no processo ensino-aprendizagem. Método é a ação pela qual são organizadas as atividades docentes e discentes para atingir objetivos pedagógicos (Libâneo, 2004). Um método ativo promove processos interativos conducentes à aprendizagem. O foco é um processo motivador e capaz de provocar mudanças na forma de aprender. Nesse contexto elegemos as metodologias ativas no processo ensino-aprendizagem.

Para Anastasiou e Alves (2004), as metodologias ativas são mecanismos por meio dos quais se aprofundam os diversos conteúdos com a finalidade de se alcançarem os objetivos de aprendizagem propostos, segundo uma determinada concepção pedagógica. Seria o mesmo que propor alcançar um objetivo pelos meios adequados.

As metodologias ativas utilizam a problematização como estratégia de ensino-aprendizagem, com vistas a motivar o discente diante de um problema. Ele se deteria, examinaria, refletiria, estabeleceria relações com a sua vivência e passaria a ressignificar suas descobertas. A problematização pode levá-lo a ter contato com determinadas informações e assim produzir conhecimento com a finalidade de solucionar impasses e promover o seu próprio desenvolvimento (Mitre et al., 2008).

Nessa concepção o discente assume um papel ativo na construção do conhecimento e o docente, um mediador/facilitador dessa construção. Desse modo, os saberes passam a ser refletidos e reelaborados pelo discente, para se constituir em conhecimento próprio (Vasconcellos, 1992). 
Essas estratégias representariam a operacionalização de alguns tipos de objetivos de aprendizagem. Neste curso, os objetivos cognitivos se operacionalizariam por meio dos grupos tutoriais, estudos de caso, estudo dirigido, seminários e miniexposições; os objetivos de habilidade, por meio do treinamento de habilidades (simulação, dramatização, observação); os objetivos atitudinais, com os grupos tutoriais, estudos de caso, simulações e dramatizações; e os objetivos mistos, com os projetos em equipe, práticas na comunidade e oficinas de trabalho.

\section{Sistema de avaliação}

Contemplamos no projeto pedagógico do curso o estudo de múltiplas áreas e dimensões das práticas em saúde, e para variadas estratégias educacionais, instrumentos de avaliação específicos. A premissa do curso é que se pode aprender com a experiência, posto que se é capaz de compreender os sentidos do vivido. O desenvolvimento da aprendizagem é bem mais amplo do que o sistema de avaliação de um curso; contudo, pensamos o sistema de avaliação também como um espaço em que se produziriam experiências de aprendizagem, tanto pela possibilidade de verificarmos a qualidade das estratégias de ensino utilizadas quanto pela própria experiência dialógica entre mestrandos e docentes, ao atribuírem valores às suas práticas de ensino e aprendizagem. Enfim, neste curso o sistema de avaliação pode ser entendido como um instrumento de sistematização, de registro e validação dos processos de aprendizagem, como também de certificação.

$\mathrm{Na}$ avaliação dos mestrandos, prioritariamente de caráter formativo, o foco é no processo de desenvolvimento de competência para atuar na ESF. Também inclui a dimensão somativa. Dada a diversidade de estratégias educacionais utilizadas para o alcance dos objetivos específicos em cada módulo, propusemos instrumentos para registro e, ao mesmo tempo, para o processo de avaliação: síntese de situações-problema processadas em grupos tutoriais, síntese das narrativas, relatório da oficina de trabalho, sínteses individuais ou coletivas de seminários, relatos da prática na comunidade, avaliação de treinamento de habilidade, registro do desenvolvimento de projeto em equipe, síntese de estudo dirigido, avaliação da simulação e síntese do estudo de caso.

$\mathrm{Na}$ avaliação formativa é possível ao estudante e docente um acompanhamento do processo ensino-aprendizagem e assim verificar se os objetivos propostos nos módulos estão sendo alcançados. Dessa forma, o mestrando, por meio de feedbacks, pode conhecer sua progressão, reavaliar seu estudo e se sentir motivado a superar dificuldades encontradas (Almeida, 1997).

Para a avaliação do curso, como ele deve ser realizado por cada nucleadora, elaboramos um questionário em formato eletrônico para ser respondido 
online ao final de cada módulo e ao final do curso, respeitando o anonimato de cada respondente, conforme os preceitos bioéticos. As informações advindas das respostas, analisadas no seu conjunto, serviriam para orientar melhorias futuras dos processos de ensino-aprendizagem.

\section{Tecendo a rede: reflexões finais}

Para nós, como formuladores da proposta do curso, alguns elementos foram determinantes: aprendizagem colaborativa, vivência e respeito à interdisciplinaridade, gestão compartilhada, troca de saberes, capacidade de romper barreiras para o uso de novas metodologias e relacionar o processo de ensino-aprendizagem ao mundo do trabalho. Destacamos nessa dinâmica a estreita relação que se pode estabelecer entre o mundo do trabalho e as discussões teórico-metodológicas realizadas, o que motivou e refinou os projetos de investigação e de intervenção, e recolocou os objetos de estudo no contexto da Atenção Primária.

Entendemos que experiências como essa devem ser compartilhadas, por acreditarmos que elas implicam um maior compromisso social o qual o grupo à frente deste projeto assumiu.

O curso obteve financiamento do Ministério da Saúde e, no final de 2011 , realizou o primeiro processo seletivo, com oferta de cem vagas e mais de 2.000 candidatos. Sinalizamos que a primeira turma encontra-se em fase de defesa das dissertações, tendo sido programada a avaliação do curso nas dimensões quantitativa e qualitativa. Na primeira dimensão, a avaliação foi realizada pelos mestrandos por meio do formulário online em que constava um conjunto de afirmativas em 11 dimensões: estrutura do curso, metodologias adotadas, desenvolvimento de competências, estratégias propostas nos módulos, material didático, estrutura física, gestão do curso, sistema de avaliação, comunicação e relações interpessoais, orientação de dissertação e processo seletivo. As respostas encontram-se em fase de análise. Na segunda dimensão, serão realizados grupos focais em todas as nucleadoras com os docentes e mestrandos, separadamente.

Já podemos registrar alguns avanços decorrentes do resultado das avaliações dos mestrandos ao término de cada módulo: permitiu a revisão permanente do material didático do curso; possibilitou maior explicitação por parte dos docentes das vantagens e desvantagens das metodologias ativas no processo de ensino-aprendizagem; e permitiu melhor distribuição da carga horária e das estratégias pedagógicas nos módulos remanescentes, entre outros aspectos.

O aperfeiçoamento do material didático do curso será publicado por uma das nucleadoras para ser divulgado a audiências mais amplas. Além disso, 
temos hoje um corpo docente capacitado e sensibilizado para processos de formação nos moldes deste aqui apresentado e a realização de estudos que tratam e refletem a realidade dos profissionais e dos seus cenários de prática.

Ao divulgarmos essa experiência que trata do desenho de um currículo no âmbito da formação stricto sensu, desejamos que outras experiências possam ser, não somente pensadas e implementadas, mas também disseminadas a públicos interessados no assunto.

\section{Colaboradores}

Maria de Fátima Antero Sousa Machado, Neiva Francenely Cunha Vieira e Virginia Alonso Hortale trabalharam na concepção e escrita do artigo. Anya Pimentel Gomes Fernandes Vieira-Meyer, Ana Patrícia Pereira Morais, Andréa Silvia Walter de Aguiar, Annatália Meneses de Amorim Gomes, Geórgia Sibele Nogueira da Silva, Liberata Campos Coimbra, Maria Socorro de Araújo Dias e Maristela Inês Osawa Vasconcelos fizeram a revisão crítica do conteúdo intelectual. Carlos Otávio Fiuza Moreira, José Batista Cisne Tomaz, Franklin Delano Soares Forte e Claudete Ferreira de Souza Monteiro revisaram o texto final.

Resumen Se trata de una reflexión sobre el proceso de desarrollo del currículo del curso de Maestría Profesional en Salud de la Familia, realizado por la Red Nordeste de Formación en Salud de la Familia. Los actores de este proceso fueron docentes de instituciones de enseñanza e investigación, federales y estatales. La construcción del currículo, con duración de dos años, incluyó la formación del Grupo de Desarrollo Curricular, responsable del diseño del curso y de la formación de sus docentes. Se discuten las etapas de este proceso teniendo como referencia las bases pedagógicas que orientaron el diseño del currículo, con énfasis en la formación de profesionales de la salud en la modalidad maestría profesional. Se espera que la divulgación de esta experiencia innovadora en el ámbito de la formación stricto sensu contribuya para que otras experiencias puedan no solamente ser pensadas e implementadas, sino también divulgadas.

Palabras clave formación en salud; diseño de currículo; salud de la familia. 


\section{Notas}

1 Universidade Regional do Cariri, Centro de Ciências Biológicas e da Saúde, Departamento de Enfermagem, Mestrado Profissional em Saúde da Família, Nucleadora URCA, Crato, Ceará, Brasil.

$<$ fatimaantero@uol.com.br>

Correspondência: Rua Coronel Antonio Luis, 1.161, Pimenta, CEP 63105-000, Crato, Ceará, Brasil.

2 Universidade Federal do Ceará, Departamento de Enfermagem, Faculdade de Farmácia, Odontologia e Enfermagem, Mestrado Profissional em Saúde da Família, Nucleadora UFC, Fortaleza, Ceará, Brasil.

$<$ neivafrancenely@hotmail.com>

3 Fundação Oswaldo Cruz, Escola Nacional de Saúde Pública Sergio Arouca, Departamento de Administração e Planejamento em Saúde, Manguinhos, Rio de Janeiro, RJ, Brasil.

<virginia@ensp.fiocruz.br>

4 Fundação Oswaldo Cruz, Mestrado Profissional em Saúde da Família, Nucleadora Fiocruz, Fortaleza, Ceará, Brasil.

<anyavieira10@gmail.com>

5 Universidade Estadual do Ceará, Centro de Ciências da Saúde, Mestrado Profissional em Saúde da Família, Nucleadora UECE, Fortaleza, Ceará, Brasil.

<anapatricia.morais@uece.br>

6 Universidade Federal do Ceará, Faculdade de Farmácia, Odontologia e Enfermagem, Mestrado Profissional em Saúde da Família, Nucleadora UFC, Fortaleza, Ceará, Brasil. <aswaguiar@yahoo.com.br>

7 Universidade Estadual do Ceará, Mestrado Profissional em Saúde da Família, Nucleadora UECE, Fortaleza, Ceará, Brasil.

$<$ annataliagomes@gmail.com>

8 Universidade Federal do Rio Grande do Norte, Centro de Ciências Humanas Letras e Artes, Departamento de Psicologia, Mestrado Profissional em Saúde da Família, Nucleadora UFRN, Natal, Rio Grande do Norte, Brasil.

<gsibele@gmail.com>

9 Universidade Federal do Maranhão, Centro de Ciências da Saúde, Departamento de Enfermagem, Mestrado Profissional em Saúde da Família, Nucleadora UFMA, São Luís, Maranhão, Brasil.

<liberatacoimbra@gmail.com>

10 Universidade Estadual Vale do Acaraú, Centro de Ciências da Saúde, Mestrado Profissional em Saúde da Família, Nucleadora UVA, Sobral, Ceará, Brasil.

<socorroad@gmail.com>

11 Universidade Estadual Vale do Acaraú, Centro de Ciências da Saúde, Mestrado Profissional em Saúde da Família, Nucleadora UVA, Sobral, Ceará, Brasil.

<miosawa@gmail.com> 
12 Fundação Oswaldo Cruz, Escola Nacional de Saúde Pública Sergio Arouca, Departamento de Ciências Sociais, Manguinhos, Rio de Janeiro, RJ, Brasil.

<otavio@ensp.fiocruz.br>

13 Secretaria de Saúde do Estado do Ceará, Escola de Saúde Pública, Fortaleza, Ceará, Brasil.

<batistatomaz@gmail.com>

14 Universidade Federal da Paraíba, Centro de Ciências da Saúde, Departamento de Clínica e Odontologia Social, Mestrado Profissional em Saúde da Família, Nucleadora UFPB, João Pessoa, Paraíba, Brasil.

$<$ fdsforte@terra.com.br $>$

15 Universidade Federal do Piauí, Centro de Ciências da Saúde, Mestardo Profissional em Saúde da Família, Nucleadora UFPI, Teresina, Piauí, Brasil.

<claudetefmonteiro@hotmail.com>

\section{Referências}

ALMEIDA, Ana M. F. P. A avaliação da aprendizagem e seus desdobramentos. Revista de Avaliação Institucional da Educação Superior, Campinas, v. 2, n. 2, p. 4, 1997.

ANASTASIOU, Léa G. C.; ALVES, Lonir P. Processos de ensinagem na universidade: pressupostos para as estratégias de trabalho em aula. 3. ed. Joinville: Univille, 2004.

BARROSO, Maria G. T.; VIEIRA, Neiva F. C.; VARELA, Zulene M. V. (Org.). Saúde da família: abordagem multirreferencial em pesquisa. Sobral: Edições UVA, 2002. 275p.

BINZ, Mara C.; MENEZES FILHO, Eliezer W.; SAUPE, Rosita. Novas tendências, velhas atitudes: as distâncias entre valores humanísticos e inter-relações observadas em um espaço docente e assistencial. Revista Brasileira de Educação Médica, Rio de Janeiro, v. 34, n. 1, p. 28-42, 2010.

BORDENAVE, Juan D.; PEREIRA, Adair M. Estratégias de ensino-aprendizagem. Petrópolis: Vozes, 1998.
BRASIL. Ministério da Educação. Portaria Normativa n. 17, de 28 de dezembro de 2009. Dispõe sobre o mestrado profissional no âmbito da Fundação Coordenação de Aperfeiçoamento de Pessoal de Nível Superior-CAPES. Diário Oficial da União. Brasília, 29 dez. 2009. Seção 1, p. 20-21. Disponível em: <www.capes. gov.br/images/stories/download/avaliacao/ avaliacao-n/Port-MEC-17-2009-mestradoprofissional.pdf>. Acesso em: 8 abr. 2014.

CECCIM, Ricardo B.; FEUERWERKER, Laura C. M. O quadrilátero da formação para a área da saúde: ensino, gestão, atenção e controle social. Physis: Revista de Saúde Coletiva, Rio de Janeiro, v. 14, n. 1, p. 41-65, 2004.

FRENK, Julio et al. Health professionals for a new century: transforming education to strengthen health systems in an interdependent world. The Lancet, Londres, v. 29, p. 5-40, 2010.

HORTALE, Virginia A. et al. Construção teórico-metodológica e aprendizados com 
a experiência no mestrado profissional em saúde da família. Trabalho, Educação e Saúde, Rio de Janeiro, v. 13, supl. 2, p. 11-23, 2015.

KELLY, Albert V. O currículo: teoria e prática. São Paulo: Harbra, 1981.

LIBÂNEO, José C. Didática. São Paulo: Cortez, 2004.

MASETTO, Marco T. Competência pedagógica do professor universitário. São Paulo: Summus, 2003.

MITRE, Sandra M. et al. Metodologias ativas de ensino-aprendizagem na formação profissional em saúde: debates atuais. Ciência \& Saúde Coletiva, Rio de Janeiro, v. 13, supl. 2, p. 2.133-2.144, 2008.

MORIN, Edgard. Os sete saberes necessários à educação do futuro. 3. ed. São Paulo: Cortez; Brasília, DF: Unesco, 2001.

SANTOS, Boaventura S. A universidade do século XXI: para uma reforma democrática e emancipatória da universidade. 3. ed. São Paulo: Cortez, 2010.

SAUPE, Rosita et al. Avaliação das competências dos recursos humanos para a consolidação do Sistema Único de Saúde no Brasil. Texto \& Contexto Enfermagem, Florianópolis, v. 16, n. 4, p. 654-61, 2007.
SMITH, Ben J.; TANG, Kwok C.; NUTBEAM, Don. WHO Health Promotion Glossary: new terms. Health Promotion International, Genebra, v. 21, n. 4, p. 340-345, 2006.

SOUZA, Nadia A. A relação teoria-prática na formação do educador. Semina: Ciências Humanas e Sociais, Londrina, v. 22, p. 5-12, set. 2001 .

TOMAZ, João B. O papel e as características do professor. In: MAMEDE, Silvia; PENAFORTE, Julio (Org.). Aprendizagem baseada em problemas: anatomia de uma nova abordagem. Fortaleza: Hucitec, 2001. p. 159-182.

VASCONCELLOS, Celso S. Metodologia dialética em sala de aula. Revista de Educação $A E C$, São Paulo, n. 83, p. 28-55, 1992.

ZAFIRIAN, Philippe. Objetivo competência: por uma nova lógica. São Paulo: Atlas, 2001.

Recebido em 16/06/2014

Aprovado em 29/03/2015 